\title{
Acoustic Detection Utility for Pump Design and Management
}

\author{
[ G. Ciaravino, C. Ciaravino ]
}

\begin{abstract}
The cavitation phenomenon determines a decrease in performance levels of hydraulic machinery together with a distinctive noise in the fluid flow. The analysis conducted on the basis of acoustic and visual measurements show that the classical method for identification of cavitation inception, based on a fall in the pump head, is not very reliable. In particular the acoustic measurement makes it possible to detect cavitation at its onset under the most potentially damaging conditions namely high values of flow rate and NPSH. Moreover the acoustic method (characterized by non intrusive technology) also makes it possible to detect different nuclei of cavitation in the pump under the same operating conditions. These characteristics of the acoustic method are of particular interest to the manufacturers of hydraulic machinery as well as to the managers of pumping systems.
\end{abstract}

Keywords-cavitation noise, centrifugal pump, cavitation detection, acoustic method.

\section{Introduction}

Centrifugal pumps are frequently subject to cavitation because of their internal geometries and/or under particular operating conditions. As a phenomenon which may lower performance levels and lead to a rapid deterioration of the pump, cavitation is an important factor demanding the attention of pump manufacturers as well as plant designers and managers, all of whom are interested in determining parameters that make it possible to detect cavitation as soon as it sets in. In technical practice the classic method for identifying cavitation, also known as the indirect method, involves determining the Net Positive Suction Head (NPSH). The NPSH indicates the absolute total head on the suction flange of the pump minus the vapour tension height tv:

$$
N P S H=z+p / \gamma+V^{2} / 2 g+p_{a t}-t_{v}
$$

where: $\mathrm{z}=$ inlet height with respect to the pump rotor plane; $\mathrm{p}=$ pressure $; \mathrm{V}=$ velocity; $\quad \mathrm{p}_{\mathrm{at}}=$ atmospheric pressure; $\gamma=$ specific weight; $g=$ acceleration of gravity.

\section{Giulio Ciaravino}

Full Professor, University of Naples Federico II, Italy

Cristiano Ciaravino

Engineer + MEng, Italy
In particular if the NPSH value falls below the NPSH3\% value, corresponding to a $3 \%$ drop in the pump head for a given flow, cavitation is triggered [1, 2, 3]. A more precautionary criterion can involve lowering the threshold to NPSH1\%. However some experiments have shown that a pump may be affected by cavitation even before the $1 \%$ drop in head $[4,5]$. Indeed, by using the acoustic method (a non intrusive technique for cavitation detection), it has been shown that in the inception phases of the phenomenon (so with the cavitation characteristic noise already detectable) an increase in the pump head is verified before the subsequent (and well known) clear decrease. A similar phenomenon has been observed (again detected by means of acoustic method) by studying the flow through a short pipe [6] during cavitation phase. In particular during the incipient cavitation phases a rise in the discharge coefficient of the short pipe was observed before an evident fall, which occurs in the advanced cavitation phase. Such phenomena might be explained with the hypothesis that, during cavitation phases, the formation of cavitating structures is able to improve the inlet of fluid threads thus causing (through lower energy fall) the temporary increase either in head (in case of pump) or in the discharge coefficient (in case of short pipe) [7]. Therefore it seems fitting to support the indirect method based on the fall in head by setting up direct methods that can detect cavitation at its inception. In this way it is possible to identify the (NPSH) values corresponding to the actual onset of cavitation.

\section{Acoustic and visual detection: cavitation curves}

Recently the experiments carried out [8] have shown how the acoustic method makes it possible to identify cavitation curves in the plane [Q, NPSH] for determining the $\mathrm{NPSH}_{\mathrm{i}}$ of actual cavitation inception. The cavitation curve is defined as the curve that subdivides the plane into points representing cavitation free conditions (above the curve) and points in which cavitation is present (below the curve). Therefore, it can be stated that the further the pump's operating point is above the cavitation curves, the safer the operating conditions are. In order to carry out experimental detection of cavitation curves for the centrifugal pump under examination, a particular plant has been used (Figure 1). It is a closed circuit system whose supply reservoir is made up of an air-tight tank (recycling tank). The centrifugal pump is positioned above the water level of the recycling tank in order to make the inception of cavitation at the rotor inlet easier. The values of the circulating flow rate are determined using a mass flow rate measuring device based on the Coriolis effect previously calibrated using a Venturi meter. 
Proc. of the Third Intl. Conf. Advances in Civil, Structural and Mechanical Engineering- CSM 2015 Copyright (C) Institute of Research Engineers and Doctors, USA .All rights reserved.

ISBN: 978-1-63248-062-0 doi: 10.15224/ 978-1-63248-062-0-60

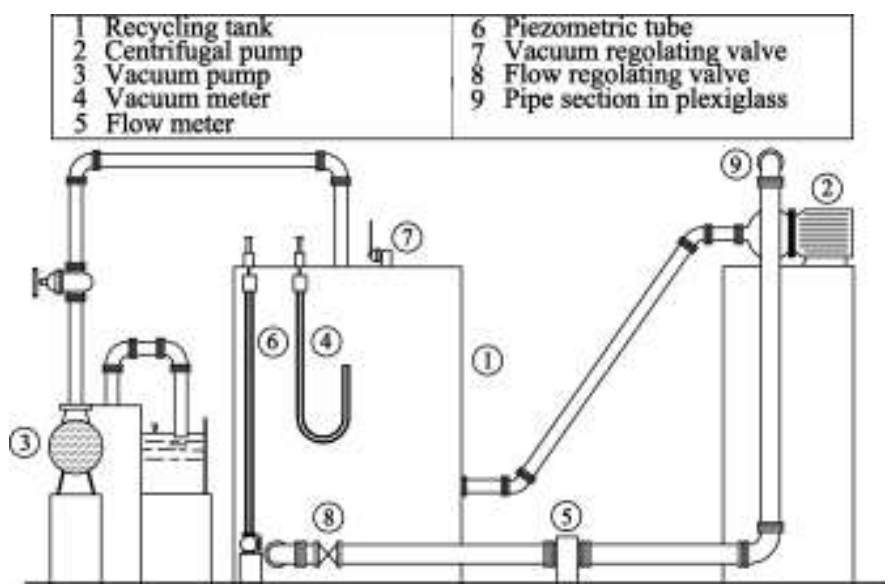

Figure 1 Experimental plant.

The system is equipped with a gate valve that makes it possible to vary the flow rate without substantially modifying the NPSH, as these variations are only related to the head loss values in the suction pipe. The system can be set to almost null absolute pressure values by means of a vacuum pump fitted to the supply tank. The tank is fitted with a vacuum meter and a piezometer to determine its internal head. Reducing the pressure in the tank varies the NPSH without substantially varying the circulating fluid flow rate, even though reducing the pressure in the tank causes the inception of cavitation which brings about a slight reduction in flow rate. The tests were carried out while keeping the circulating flow rate constant (apart from the small variations mentioned above) and reducing the pressure in the tank until flow is interrupted. For each test carried out using the above described measurement equipment, it is possible to determine the values for flow rate $\mathrm{Q}$, head in the tank, head in the suction pipe, head in the delivery pipe, pump discharge head and NPSH. In order to achieve a complete observation of the phenomenon acoustic and visual measurements were taken during each test. Indeed, since in the field of lower flow rates the acoustic measurements seems to be less efficient, a comparison was made using the visual measurements. Visual measurements are possible because the experimental installation is endowed with a section of plexiglass pipe immediately downstream of the pump. As far as the acoustic measurements are concerned, it was observed that during each test series, a fall in pressure in the tank modifies the characteristics of the total noise due to cavitation, produced by the system in the suction pipe. The acoustic measurements were taken (within the respective limits) both directly by the human ear and using a phonometer with microphone and a signal digital analyser. In these tests, the noise emitted by the phenomenon for particular frequencies was not filtered out. The total noise modifications for all tests always occurred with the same order for each type (described later) and they disappeared when the flow was interrupted. In short, for every flow rate and as the pressure in the supply reservoir decreases, five types of acoustic effects were identified. They have been classified in the order in which they occur as follows:

$$
\begin{array}{ll}
- & \text { discontinuous crackling (C.50\%); } \\
\text { - } & \text { continuous crackling (C.C.); } \\
\text { - } & \text { discontinuous hissing (S.50\%); } \\
\text { - } & \text { continuous hissing (C.S.); } \\
\text { - } & \text { deep noise (D.N.). }
\end{array}
$$

The visual observations can also be divided, for every flow rate and as pressure falls, into two different types that have been classified in the order in which they occur as follows:

- $\quad$ small bubbles (S.B.);

- $\quad$ limit bubbles (L.B.).

The diagram in Figure 2 reports, as functions of the flow rate $\mathrm{Q}$ and the NPSH, the points representing the various acoustic and visual measurements.

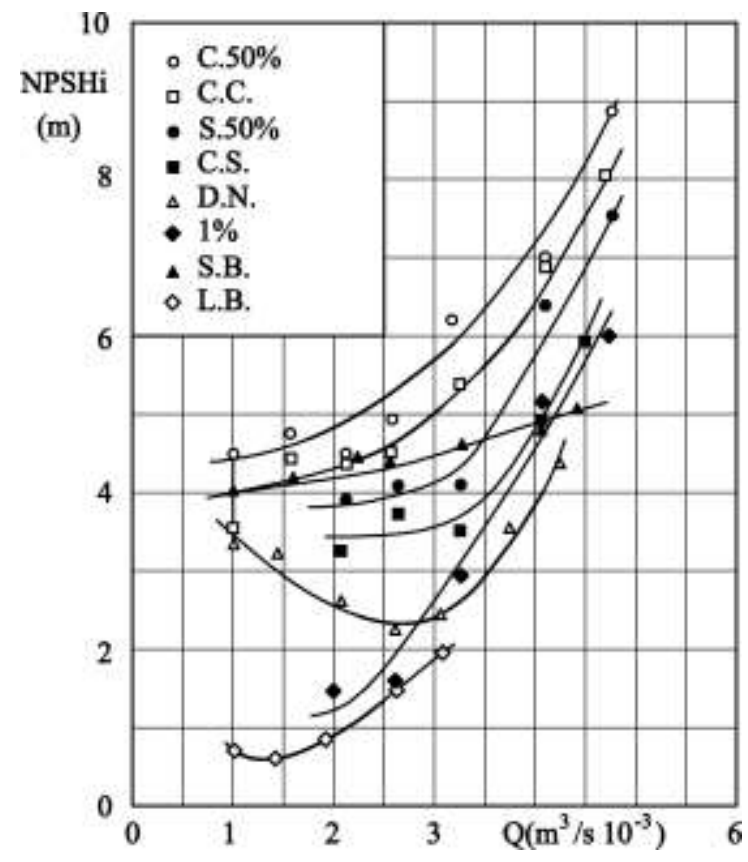

Figure 2 Cavitation curves.

In particular the diagram in Figure 2 plots the interpolating curves for each of the different type of acoustic and visual detection. These curves can be considered as cavitation curves for that particular type of detection. In other words, each single curve, for that particular type of acoustic or visual measurement, subdivides the plane (as already mentioned) into points in which cavitation is absent (above the curve) and points in which cavitation is present (below the curve). Therefore, it can be stated that the further the pump's operating point is above the cavitation curves, the safer the operating conditions. 


\section{Discussion on cavitation curves}

The experimental tests carried out show that the acoustic measurement types (crackling, hissing, etc.) taken for each flow rate always occur in the same order reported above. It can also be observed that the swarm of small bubbles appears in relation with several types of acoustic effect associated with the varying flow rates: in particular a deep noise, in the case of high flow rates, and a discontinuous hissing, in the case of low flow rates. Moreover limit bubbles appear just before flow detachment and at very low NPSH values: in particular the lower the flow rates, the smaller the NPSH values. The diagram in Figure 2 also reports the $1 \%$ cavitation curve, obtained from the measurements. We can see that the $1 \%$ curve remains below almost all the operating conditions. This observation confirms that referring to the NPSH1\% (or NPSH3\%) parameter in order to identify the inception of cavitation is not a very reliable technique. Furthermore, for values of NPSH that are not particularly low (NPSH $=4 \div 5 \mathrm{~m}$ ) cavitation is already present with swarms of small bubbles. The trend of the cavitation curves shows that the system better resists cavitation (and its related drawbacks) at low flow rates, where the acoustic measurement is not very efficient. The existence of working conditions in which cavitation is not effectively detected by means of the acoustic method (but it is detected through visual method) could find an explanation through the following observations. First of all the existence of cavitating bubbles detected in the transparent pipe section placed downstream of the pump was observed with reference to low values of flow rate and, therefore, of bubble velocity. Under such conditions high permanency periods allow bubble growth (through absorbption of vapour by evaporation of surrounding liquid) and bubble unification by constituting larger bubbles filled with vapour. Such larger bubbles (limit bubbles) have longer reabsorption times in the surrounding liquid (when favourable pressure conditions occur) than small bubbles thus determining the following two conditions: (i) bubbles are still evident in the pump outlet, (ii) the characteristic cavitation noise is not determined because a quick implosion of bubbles does not occur. It should be pointed out that in spite of the circumstances above mentioned the acoustic detection method seems to be particularly sensitive and useful in the most potentially damaging fields for the pump, corresponding to higher flow rates and NPSH values.

\section{Measurement utility with the acoustic method}

Cavitation effectively causes profound modifications in the structure of the acoustic signal: the experimental measurements point out the presence of a marked increase in the spectral power density curve of noise signal especially in the high frequency range $[9,10,11,12,5,6]$. Within certain limits, the modifications to the acoustic signal can also be perceived directly by the human ear; for a complete analysis, it is necessary to use instrumentation ensuring no direct interaction with either the fluid stream or the machinery under examination (unlike the other direct detection methods). In a series of tests (reported in Table 1) conducted on a simplified experimental plant (schematically shown in Figure 3) in which background noise is considerably reduced and experimental data were collected by means of essential but precise and reliable equipment (mercury manometer and an accurately calibrated Venturi flume).

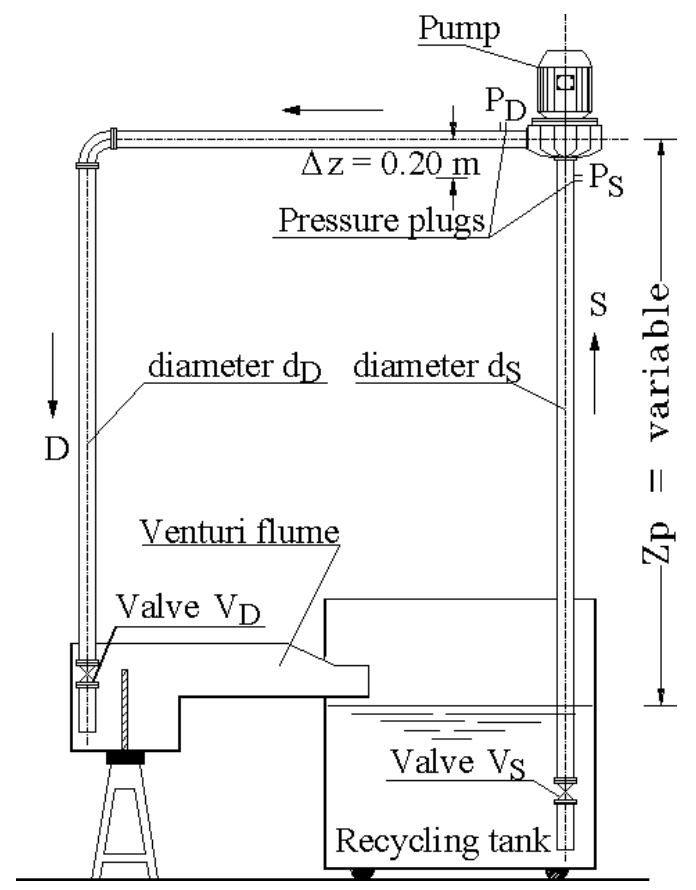

Figure 3 Experimental plant.

The suction pipe and delivery pipe have different diameters $\left(\mathrm{d}_{\mathrm{S}}=0.0415 \mathrm{~m}, \mathrm{~d}_{\mathrm{D}}=0.0335 \mathrm{~m}\right.$ respectively $)$ as well as regulating valves; the pump (schematically shown in Figure 4, normally operating at 1410 r.p.m. and having a maximum head of about $11 \mathrm{~m}$ ) is placed at a height $Z_{p}$ which may be changed if compared to the water level in an open recycling tank $\left(Z_{p}=1.95 \mathrm{~m}\right.$ in the test reported in Table 1$)$.

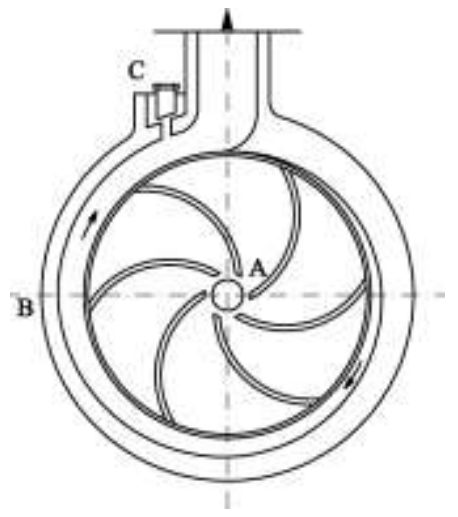

Figure 4 Scheme of pump. 
In the acoustic measurements of the tests carried out on the pump, a phonometer with microphone and an overall $22.5 \mathrm{kHz}$ passband was used. The signal picked up by the phonometer was sent to a $1 / 3$ octave real time spectrum analyser which provided the histogram of the spectral power densities for the various frequencies investigated.

The tests highlighted some notable results:

the method is highly sensitive and the acoustic effects of cavitation are manifested in the examined conditions for NPSH values that may be very high (more than $7 \mathrm{~m}$ );

the method can detect not only the presence of cavitation but also its location by analysing the histogram of the spectral power densities.

Table 1 Experimental tests.

\begin{tabular}{|c|c|c|c|c|c|c|c|}
\hline Test & $\left(\mathrm{m}^{3} \mathrm{~s}^{-\frac{Q}{1}}-0^{3}\right)$ & $\begin{array}{l}P_{x y} \\
\text { (im) }\end{array}$ & $\begin{array}{l}P_{D \gamma} \gamma \\
\text { (m) }\end{array}$ & $\begin{array}{c}\mathrm{r}_{s}^{2} 2 \mathrm{gg} \\
\text { (m) }\end{array}$ & $\begin{array}{c}V_{b}^{2} 2 g \\
(\mathrm{~m})\end{array}$ & $\begin{array}{l}H_{p} \\
\text { (m) }\end{array}$ & $\begin{array}{c}\text { NPSH } \\
\text { (m) }\end{array}$ \\
\hline 1 & 1.10 & -1.84 & 8.61 & 0.03 & 0.68 & 10.70 & 8.12 \\
\hline 2 & 1.28 & -187 & 8.50 & 1).05 & 0.11 & 10.63 & 811 \\
\hline 3 & 1.33 & -1.88 & $8+6$ & 0.05 & 0.12 & 10.61 & 8.10 \\
\hline 4 & 1.75 & -1.95 & 8.03 & 0.09 & (1) 20 & 10.29 & 8.07 \\
\hline 5 & 200 & -2.03 & 7.62 & 0.12 & 1) 29 & 10.02 & 802 \\
\hline 6 & 238 & -2.11 & 7.15 & 10. 16 & 0.37 & 9.67 & 798 \\
\hline 7 & 2.62 & $-2,17$ & 6.70 & 6. 19 & 0.45 & 933 & 795 \\
\hline 8 & 2.76 & -2.21 & $6 .+4$ & $10 / 21$ & 0.50 & 9.14 & 793 \\
\hline 9 & 2.96 & -2.26 & 6.15 & 0.24 & 057 & 892 & 7.91 \\
\hline 10 & 3.03 & $-2,31$ & 584 & 0.26 & 0.60 & 8.62 & $7 \mathrm{s8}$ \\
\hline 11 & 3.29 & -2.39 & 5.16 & 10.30 & 0.71 & X.16 & 784 \\
\hline 12 & 3.48 & $-2,47$ & 464 & 10,34 & $0.7 y$ & 7.76 & 780 \\
\hline 13 & 3.69 & -2.55 & 3.49 & 1).38 & 0.89 & 752 & 7.76 \\
\hline 14 & 3.80 & -2.65 & 3.46 & (2. 40 & 095 & $6 .+8$ & 7.70 \\
\hline 15 & 396 & $-2,65$ & 294 & 0. 44 & 103 & 6.38 & 7.72 \\
\hline 16 & +.04 & -2.68 & 2.71 & 0.45 & 1.07 & 6.21 & 7.70 \\
\hline 17 & 409 & $=2.73$ & 2.39 & (1). 47 & 1010 & 5.95 & 767 \\
\hline 18 & 4.12 & -2.76 & 2.13 & 0. 47 & 1.11 & 5.73 & 7.64 \\
\hline 19 & 4.201 & -2.84 & $2(x)$ & $0+49$ & 1.16 & 567 & 7.58 \\
\hline 20 & +21 & -2.78 & 213 & 1) 49 & 1.16 & 5.55 & 764 \\
\hline 21 & 4.22 & -2.85 & 186 & 0.50 & 1.17 & 555 & 7.58 \\
\hline 22 & 425 & -2.86 & 1.74 & 4) 50 & 1.19 & 5.45 & 757 \\
\hline 23 & +29 & -2.78 & 1.65 & 0.51 & 1.21 & 5.31 & 7.66 \\
\hline 24 & 4.35 & $-2,81$ & 1.35 & 0.53 & 1.24 & 5.07 & 7.65 \\
\hline 25 & 4.40 & -2.94 & 109 & 0.54 & 1.27 & 492 & 7.53 \\
\hline 26 & ++1 & -2.84 & 1.16 & 1). 54 & 128 & 424 & 7.63 \\
\hline 27 & $4+7$ & -2.85 & 0.98 & (1) 56 & 1.31 & 479 & 764 \\
\hline 28 & 4.48 & -2.89 & (1) 82 & 10.56 & 132 & 4.67 & 7.60 \\
\hline 29 & 4.54 & -2.92 & 0.62 & 0.57 & 1,35 & 452 & 7.58 \\
\hline 30 & 4.56 & -3.02 & 028 & 0.58 & 136 & 428 & $7+49$ \\
\hline
\end{tabular}

These results can be inferred from Table 1 and from the histograms reported in Figures 5, 6 and 7. For tests with increasing flow rate $\mathrm{Q}$ and for the suction pipe and delivery pipe respectively, are reported in Table 1: piezometric height values $\mathrm{P}_{\mathrm{S}} / \gamma$ (piezometric inlet set to $-0.20 \mathrm{~m}$ referred to the pump rotor plane, see Figure 3 ) and $\mathrm{P}_{\mathrm{D}} / \gamma$; kinetic height values $\mathrm{V}^{2}{ }_{\mathrm{S}} / 2 \mathrm{~g}$ and $\mathrm{V}_{\mathrm{D}}^{2} / 2 \mathrm{~g}$; the corresponding values of delivery head $\mathrm{H}_{\mathrm{P}}$ and of NPSH. Figures 5, 6 and 7 report the histograms of some tests for larger flow rates present in Table 1 and specifically for Tests $\mathrm{n}^{\circ} 19,21,22,25$ and 30 . These tests were selected with the criterion, on the one hand, of verifying more developed cavitation conditions (Tests $\mathrm{n}^{\circ} 25$ and 30) and, on the other, of concentrating on the range of flow rates in which the inception of cavitation seemed to lie (Tests $n^{\circ} 19$,
21 and 22). Figures 5, 6 and 7 report the frequency $(\mathrm{kHz})$ on the horizontal axis and the noise intensity $(\mathrm{dB})$ on a distinct vertical axis for each of the five above mentioned tests $(\mathrm{I}-\mathrm{V})$.

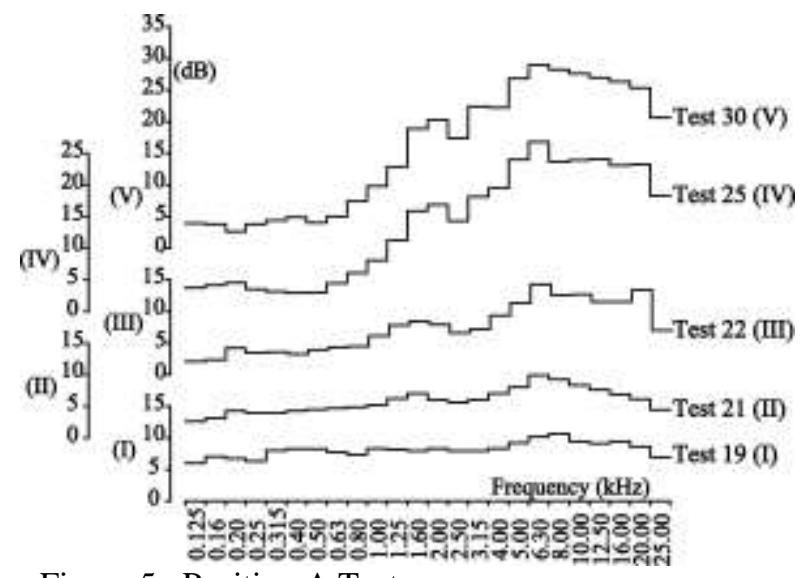

Figure 5 Position A Tests.

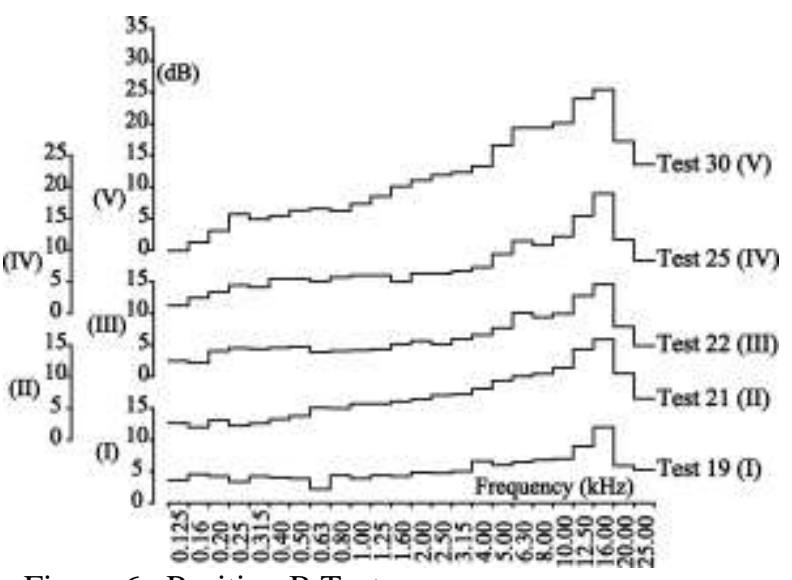

Figure 6 Position B Tests.

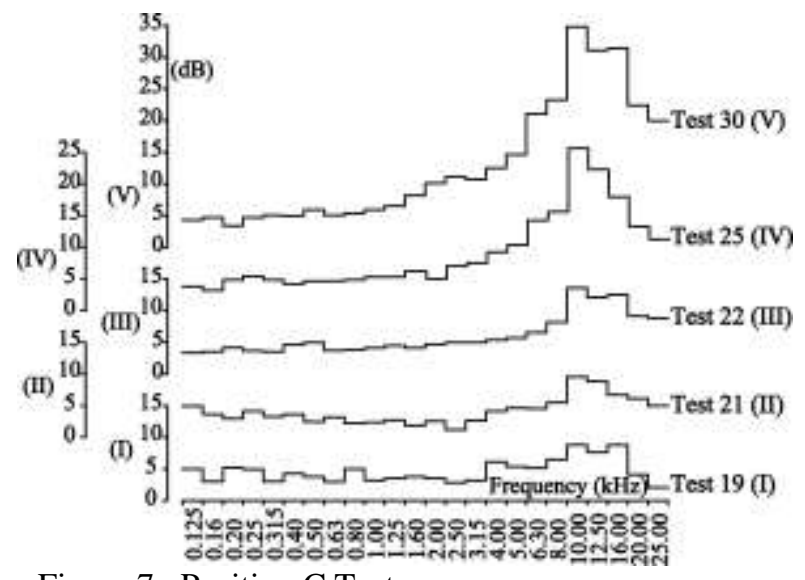

Figure 7 Position C Tests. 


\section{v. Discussion on a particular use of the acoustic method}

The difference between the three figures 5, 6 and 7 lies in the fact that the spectral power density histograms for these five tests refer to three different positions (outside the pump) of the detector microphone of the phonometer. In particular Figure 5 refers to acoustic measurements taken in position A indicated in Figure 4, while Figures 6 and 7 refer to positions $\mathrm{B}$ and $\mathrm{C}$ indicated again in Figure 4. It should be noted that in order to facilitate measurement of increases in the diagrams due to cavitation, it is not the real value of the noise intensity expressed in $\mathrm{dB}$ that is reported, but rather the difference, again expressed in $\mathrm{dB}$, between the intensity of noise emitted under test conditions and that of the so-called background noise with the same frequency. Identifying the background noise makes it possible to eliminate parasite noise from the measured signal, such as that generated by the surrounding environment and the mechanical noise emitted by the pump. In each of Figures 5, 6 and 7, Test $n^{\circ} 19$ presents a spectral power density slope that is basically flat, thus indicating the absence of high frequencies that are present in the cavitation phenomenon. From Test $\mathrm{n}^{\circ} 21$ on, it can be increasingly observed that there is a clear rise in the diagrams. While for position A (Fig. 4) this increase corresponds above all to the frequency of $6.3 \mathrm{kHz}$, for positions B (Fig. 6) and C (Fig. 7) this raise takes place at frequencies of $16.0 \mathrm{kHz}$ and $9.6 \mathrm{kHz}$ respectively. The marked difference between these three frequencies suggests that there are three different cavitation nuclei. In addition to a first nucleus located near the suction flange (position A), in the position where cavitation is expected to occur, two other unexpected cavitation nuclei were detected. These two nuclei are detected in the pump flow delivery section, in particular in the rotor spiral volute (position B) close to a bleeder orifice fitted on the delivery section of the pump casing (position C). The acoustic measurements have shown how the design and internal geometry of machinery affects the fluid flows and can thus facilitate cavitation even in areas where cavitation would not normally be expected. This selectivity, which the other methods cited do not possess and which make it possible to identify the various nuclei of cavitation in the machinery operating under the same conditions, can be of remarkable importance for pump manufacturers both in the design and in the production phase.

\section{vi. Conclusions}

The conditions of cavitation corresponding to higher flow rates and NPSH values are the conditions for which pumps are exposed to the largest damages. In this field (characterized by high frequencies) the acoustic method shows a particular sensitivity since it is suitable for detecting the phases of cavitation onset (NPSHi) before of the pump head fall (NPSH1\% or NPSH3\%). Unlike other methods for the direct detection of cavitation, the acoustic method also makes it possible to detect different nuclei of cavitation in the pump in the same working conditions. Indeed experimental tests point out that different cavitation nuclei, produced by particular hydrodynamic conditions or by particular internal pump geometries, have a their own characteristic frequency in the spectral power density histogram. The acoustic measurements also show how the design and internal geometry of machinery affects the fluid flows and can thus facilitate cavitation even in areas where cavitation would not normally be expected. This possibility of detecting different nuclei of cavitation, under particular flow conditions or particular conformations of machine geometry, can be exploited by the manufacturers of pumps both for testing prototypes in the design phase and for quality control during the manufacturing process. The characteristics of the acoustic method are of particular interest also to the managers of pumping systems. In fact the acoustic measurement equipment is non intrusive and with last generation technologies it is possible to provide a mobile detection station constituted by a phonometer and a portable and highly sensitive recording device. Such equipment is wellsuited to be used for measurements in existing pumping systems in order to verify whether the working conditions, chosen during the management phase, can trigger cavitation.

\section{References}

[1] A. C. Gongwer, "A theory of cavitation flow in centrifugal-pump impellers", Transaction of ASME, Vol. 63, pp. 29-40, 1941.

[2] E. Grist, "1974, Net Positive Suction Head requirements for avoidance of unacceptable cavitation erosion in centrifugal pump", Paper C 16374, Proceedings IMechE Conference on Cavitation, Heriot-Watt University, Edinburgh, Scotland, 1974.

[3] B. Schiavello, F.C. Visser, "Pump cavitation - varius NPSHR criteria, NPSHA margins and impeller live expectancy", Twenty Fourth International Pump Users Symposium, pp. 1-37, 2008.

[4] G. Federici, E. Reiteri and F. Siccardi, "The determination of cavitation inception in a centrifugal pump through analysis of pressure pulsation generated by the machinery", L'Energia Elettrica XLVIII (10), 1971. (In Italian).

[5] G. Ciaravino, G. Pulci Doria, "Rilievo con il metodo acustico ed interpretazione dei fenomeni di inizio cavitazione in pompe centrifughe", Idrotecnica n.3 maggio-giugno, pp. 127-140.(In Italian), 1983.

[6] G. Ciaravino, G. Pulci Doria, "The use of the laser anemometer in cavitation currents: experiments at the inlet to a short pipe", International Symposium on Application of Laser-Doppler Anemometry to Fluid Mechanics, Lisbon, July 5-7, 1982(b),.

[7] G. Ciaravino, "Analysis of particular experimental measurements in cavitating stream flow",. C. A. Brebbia, G. M. Carlomagno Eds, Computational Methods and Experimental Measurement XII, vol. 41, pp. 829-838, Southampton (UK), 2005.

[8] G. Ciaravino, "Identification of cavitation NPSH in centrifugal pump", First Joint Conference IAHR-IWA Pumps, Electromechanical Device and Systems Applied to Urban Water Management, Cabrera \& Cabrera Jr. Eds, Swets \& Zeitlinger, Lisse, pp. 525-531, 2003.

[9] F. Numachi, "Ultrasonic shock waves emitted by cavitation", XIII Congress of IAHR, pp.85-120, Kyoto, August-September, 1969.

[10] J. Varga, G. Sebestyen and A. Fay, "Detection of cavitation by acoustic and vibration measurement methods", La Houille Blanche n.2, 1969.

[11] L. Briancon-Marjollet, "Qualification acoustique de la cavitation à bulles", La Houille Blanche n.7/8, 1992.

[12] B. Stoffel, W. Schuller, "Investigations in respect of the determination of cavitation intensity by the means of acoustic signals", La Houille Blanche n.7/8, 1992. 